Colonia", en: Ma Elena Laurnaga (coord.) La geografía de un cambio: Política, Gobierno y Gestión Municipal en Uruguay. Ediciones Banda Oriental, Instituto de Ciencia Política. Montevideo.

Vairo, Daniela. (2008). "Juntos pero no casados: Los efectos de la reforma constitucional al interior de los partidos", en Revista Uruguaya de Ciencia Política No 17. pp. 159182. Ediciones Cauce - Instituto de Ciencia Política, Montevideo.

\title{
Prensa, Internet
}

Bottinelli, Oscar A (2010). "9 de mayo: se desató una tempestad cuando se pronosticaba calma". En radio El Espectador, programa En Perspectiva, espacio Análisis político, 14.05.2010.

\section{¿Protesta o cambio institucional?}

Determinantes del voto nulo y blanco en las elecciones departamentales de 2010 en Uruguay en perspectiva comparada

Gerardo Isaac Cisneros Yescas

Martín Freigedo Peláez

\section{Resumen}

Las elecciones departamentales de 2010 en Uruguay arrojaron resultados anómalos para los estándares del país, los cuales están asociadas a un significativo aumento 
del voto nulo y blanco. Este artículo busca explicar los factores que propiciaron este fenómeno en perspectiva comparada con las elecciones de 2005, partiendo de la hipótesis de que dicho aumento está asociado a la modificación en el cambio institucional debido a la incorporación en elecciones concurrentes de un nuevo nivel de gobierno (municipios). A partir de cuatro modelos econometricos, los hallazgos demuestran que para el 2005 el voto inválido está asociado positivamente con la escolaridad lo que refleja un voto de protesta; mientras que en 2010 muestra que están asociados al diseño institucional. Por su parte, cuando se analizan los votos inválidos por separado (blanco y nulo) para las elecciones del 2010, los primeros están asociados a la protesta política y los últimos al cambio institucional.

Palabras claves: voto inválido - cambio institucional - elecciones subnacionales Uruguay

\begin{abstract}
Abtract
In the 2010 departmental elections in Uruguay, the results show a significant increase of the null and blank votes. This article analyses the factors that explains this phenomenon in comparative perspective to the 2005 elections. The main hypothesis holds that the increase in null and blank votes is associated with the change in institutional design due to incorporation, in concurrent elections, of a new government level (municipalities). From four econometric models, the findings shows that in the 2005 departamental elections invalid vote is positively associated with schooling reflecting a protest vote, while in 2010 the association is with institutional design. On the other hand, when the invalid vote is analyzed separately (blank and null) for the 2010 elections, the former are associated with the political protest and the second with institutional change.
\end{abstract}

Keywords: invalid vote - institucional change - subantional election - Uruguay

\title{
I. INTRODUCCIÓN
}

El comportamiento electoral ha sido estudiado ampliamente en la literatura, sin embargo el voto inválido ${ }^{74}$ no ha estado en el foco principal de atención de los politólogos ni de los estudiosos de los regímenes sub nacionales. Por tanto, es posible identificar una carencia de investigaciones y aproximaciones sobre el tema debido a que no es un fenómeno sistemático y recurrente, presentándose por lo general de forma aleatoria y respondiendo a diversas causas.

\footnotetext{
${ }^{74}$ Por voto inválido se entiende tanto a los votos en blanco como a los nulos. En Uruguay el voto nulo y blanco se registran de forma separada brindando la posibilidad de analizarlos de manera separada como se realiza en este artículo.
} 
Siendo el voto el componente distintivo y la institución fundamental de la democracia procedimental, el registro de altos niveles de votos nulos y blancos en las elecciones es un elemento llamativo. Además, es importante tenerlo en cuenta, dado que cuando se ejerce de manera voluntaria es un claro signo de protesta y de rechazo político que puede deberse en ocasiones a un desalineamiento de los electores con relación a los partidos políticos o a un castigo por su mal desempeño. Sin embargo, también pueden presentarse otros factores que no manifiesten una acción de protesta pero que afecten el aumento de los votos inválidos, por ejemplo, las modificaciones en el marco institucional de las elecciones.

En mayo de 2010 se llevaron a cabo por tercera vez en Uruguay las elecciones de gobiernos departamentales (correspondiente al segundo nivel de gobierno) separadas de las elecciones del gobierno nacional. Asimismo, por primera ocasión, luego de la aprobación en 2010 de la Ley de Descentralización y Participación Ciudadana que creó a los municipios como tercer nivel de gobierno, se votaron en conjunto autoridades departamentales y municipales ${ }^{75}$. El resultado en este acto electoral tuvo consecuencias anómalas para los estándares uruguayos. Las anomalías estuvieron asociadas al significativo aumento del porcentaje de votos inválidos en las elecciones departamentales de 2010, pues, mientras que el promedio de este tipo de votos era de 3 por ciento en 2000 y 2005, en 2010 este porcentaje aumentó a 7.7 por ciento. Por tanto la pregunta que guía este artículo es: ¿qué factores propiciaron el aumento del voto nulo y blanco en las elecciones departamentales en Uruguay en 2010? Ante ello, el objetivo fundamental del documento es explicar las causas del aumento de dicho nivel de sufragios, comparando el comportamiento electoral de las elecciones departamentales del 2005 con las de 2010.

Para este cometido el artículo se divide en cinco secciones. En la primera se analizan de manera sintética los principales resultados de las elecciones departamentales de 2010 en comparación con las anteriores contiendas electorales donde se enfatiza el aumento de los votos blancos y anulados y, a su vez, se exponen las principales características del diseño institucional-electoral departamental y municipal.

En la segunda sección se presentan los principales enfoques que han explicado el fenómeno del voto inválido: socioeconómico, institucional y político, acentuando su importancia para la explicación del caso. Además, se retoma también la literatura sobre desalineamiento electoral, ya que el voto nulo es un signo relevante de este

75 Antes de estas elecciones de 2010, los ciudadanos solamente elegían autoridades sub nacionales en el segundo nivel de gobierno. Sin embargo, desde la inclusión de la mencionada Ley y la creación de los Municipios, se eligieron autoridades departamentales y municipales. Más adelante se profundizará en este tema. 
fenómeno; y, de igual manera, se hace hincapié en los posibles efectos que el cambio en el diseño institucional pudo generar en dicho fenómeno.

En la tercera parte se plantea el marco teórico del cual se derivan un conjunto de hipótesis explicativas del problema, atendiendo a las particularidades que presenta el caso uruguayo. Así se resalta la fuerza del cambio institucional en el aumento de los votos nulos y blancos, pues se considera que el diseño institucional afecta el comportamiento de los votantes. Asimismo, no se descuida el factor de protesta inherente a este tipo de problemáticas.

En la sección posterior se realiza el análisis empírico empleando técnicas econométricas con base en cuatro modelos de regresión lineal robusta los cuales sugieren que, a diferencia de las elecciones de 2005, en las elecciones departamentales de 2010 hubo un voto de protesta significativo debido a que los sectores con mayores recursos -es decir, los más escolarizados y de altos ingresos a nivel departamental- estuvieron asociados positivamente con el nivel de sufragios inválidos. Además, se halló evidencia que sostiene que, en 2010, al desagregar los votos inválidos en nulos y blancos, la introducción de un nuevo nivel de gobierno produjo un aumento en el porcentaje de ambos tipos de sufragio. Finalmente, se avanzan una serie de conclusiones que buscan aportar al estudio de un campo que aún está en expansión.

\section{LOS RESULTADOS ELECTORALES DEL 2010 A NIVEL DEPARTAMENTAL}

Las elecciones departamentales de mayo del 2010 arrojaron resultados inusuales para la democracia uruguaya. Nunca antes, desde la instauración del voto obligatorio en el país, se habían registrado niveles tan altos de voto inválido como en las pasadas elecciones a nivel sub nacional. Históricamente, estos votos han oscilado en promedio en el tres por ciento como puede verse en el Cuadro 1, incluso con la reforma constitucional de 1997 que modificó el sistema electoral y determinó la separación entre elecciones nacionales y departamentales, no se registraron valores tan altos de votos nulos y blancos en la elección de 2000 ni en la de 2005.

En 2010 los votos en blanco y anulados alcanzaron el 7.7 por ciento del total, lo cual representó un 79.06 por ciento de aumento con respecto al promedio de elecciones pasadas, pues en mayo del 2000 estos votos alcanzaron el 3.1 por ciento y en el 2005 el porcentaje fue de 3.7 por ciento.

Ahora, si se analizan estos resultados por departamento, los datos son todavía más interesantes ya que en todos los casos el patrón general mostró un incremento del voto nulo y blanco. Los porcentajes más altos se presentaron en Montevideo, Canelones y Cerro Largo donde hubo un aumento de más del 200 por ciento. Por otro lado, algunos departamentos no tuvieron un incremento tan estrepitoso como el señalado pero aún así se presentaron cambios importantes, por ejemplo en 
Colonia, Paysandú y Flores el aumento estuvo por encima del 100 por ciento, en tanto que en Treinta y Tres, Durazno y Salto se incrementó en 30 por ciento ${ }^{76}$.

\section{Cuadro 1: Promedio de votos nulos y blancos a nivel departamental}

\begin{tabular}{|l|l|l|l|l|l||}
\hline \hline DEPARTAMENTO & 2000 & 2005 & 2010 & $\begin{array}{l}\text { Promedio 2000 } \\
\text { y 2005 }\end{array}$ & $\begin{array}{l}\text { Diferencia } \\
\text { promedio de 2000 y } \\
2005 \text { respecto a 2010 }\end{array}$ \\
\hline \hline MONTEVIDEO & 3.25 & 3.96 & 13.77 & 3.60 & 282.07 \\
\hline CERRO LARGO & 2.37 & 2.31 & 7.61 & 2.34 & 225.28 \\
\hline CANELONES & 3.67 & 5.16 & 13.39 & 4.42 & 203.25 \\
\hline MALDONADO & 2.79 & 2.78 & 7.78 & 2.79 & 179.15 \\
\hline FLORES & 2.11 & 2.27 & 6.00 & 2.19 & 173.82 \\
\hline SORIANO & 2.71 & 3.46 & 7.33 & 3.09 & 137.55 \\
\hline ARTIGAS & 2.30 & 2.50 & 5.62 & 2.40 & 134.08 \\
\hline PAYSANDÚ & 2.44 & 2.53 & 5.62 & 2.48 & 126.21 \\
\hline SAN JOSÉ & 2.65 & 3.39 & 6.70 & 3.02 & 121.94 \\
\hline ROCHA & 3.26 & 4.58 & 8.69 & 3.92 & 121.75 \\
\hline COLONIA & 3.79 & 3.66 & 8.05 & 3.72 & 116.15 \\
\hline TACUAREMBÓ & 2.06 & 2.46 & 4.38 & 2.26 & 93.86 \\
\hline FLORIDA & 2.68 & 3.29 & 5.45 & 2.99 & 82.55 \\
\hline RIVERA & 3.09 & 2.93 & 5.20 & 3.01 & 72.77 \\
\hline RÍO NEGRO & 3.56 & 3.03 & 5.37 & 3.29 & 63.06 \\
\hline LAVALLEJA & 3.74 & 3.30 & 5.69 & 3.52 & 61.73 \\
\hline SALTO & 2.82 & 2.76 & 4.45 & 2.79 & 59.39 \\
\hline DURAZNO & 3.45 & 3.17 & 5.10 & 3.31 & 54.15 \\
\hline TREINTA \\
TRES
\end{tabular}

Más allá de estos resultados, un elemento a destacar en estas elecciones fue la diferencia en la composición del marco institucional en comparación con las

${ }^{76}$ Un punto aparte es el análisis de los resultados a nivel municipal. Siendo las primeras elecciones en las que se eligieron alcaldes y concejales a nivel local, el resultado era realmente incierto. Si bien los resultados reflejaron que no existió en todo el país la misma predisposición del votante a participar en la elección de las nuevas autoridades locales, la abstención fue por demás significativa en el total de los casos. 
elecciones de 2000 y de 2005, producto de la introducción de la llamada ley de "Descentralización y Participación Ciudadana" aplicada sólo para los comicios de 2010.

Pero, antes de pasar a ello, es necesario un poco de contexto para entender la importancia del cambio instucional en esta última elección. En primer lugar se debe aclarar que en los comicios departamentales los ciudadanos eligen por medio del voto directo al intendente y a los ediles que conforman la Junta Departamental. El intendente es elegido por mayoría simple, en tanto que la Junta Departamental por representación proporcional. Este sistema de elección es igual en los diecinueve departamentos del país.

Por otro lado, algunas de las modificaciones más significativas al marco institucional fueron resultado de la reforma constitucional de 1997, que además de una serie importante de implicaciones para el sistema electoral nacional ${ }^{77}$, también cambió el diseño institucional departamental en tres sentidos: "1) Separación en el tiempo de las elecciones departamentales respecto a las nacionales; 2) Restricción del doble voto simultáneo limitando a tres el número de candidatos a intendente por lema y; 3) La elección de convenciones departamentales." (Cardarello y Magri, 2010).

El hecho de separar las elecciones nacionales de las departamentales le otorgó a las últimas mayor autonomía, ya que antes los comicios estaban altamente condicionados por lo que aconteciera a nivel nacional, sobre todo por el fuerte protagonismo de la elección del Presidente de la República. López (2007: 98) demuestra esta relación $y$, establece que desde la separación entre ambas elecciones "(...) se ha detectado que un porcentaje muy importante de los electores a nivel departamental cambia su voto con relación a los comicios nacionales".

Esta autonomía también se ve reflejada en los resultados de las elecciones de 2010 donde la elección nacional alcanzó un porcentaje de votos inválidos de 2.8 por ciento, mientras que en los comicios departamentales, como se señaló, el porcentaje fue de 7.7 por ciento ${ }^{78}$.

El otro gran cambio que ha sufrido el marco institucional y, el cual es de gran importancia para el argumento expuesto aquí, fue el que repercutió únicamente en la elección departamental de 2010. En estos comicios se incorporaron las elecciones a nivel municipal lo que trajo consigo que los ciudadanos eligieran por medio del voto directo a un alcalde y cinco concejales por municipio. Este cambio fue producto de la mencionada ley de "Descentralización y Participación Ciudadana" que fue aprobada en el 2010, creando municipios en las localidades de más de 5000

77 Se incorporó la segunda vuelta para la elección de presidente.

${ }^{78}$ El 2.8 por ciento de la elección nacional fue calculado sumando los votos en blanco, los votos nulos y los sobre con hojas que sólo contenían un voto por el Sí, en referencia al plebiscito convocado para anular la Ley de Caducidad. 
habitantes, estableciéndose 89 alcaldías en todo el territorio nacional. Por lo tanto, no todos los ciudadanos tuvieron la oportunidad de votar por autoridades locales ${ }^{79}$, pero, en los lugares donde sí pudieron, los comicios municipales se celebraron junto con los departamentales provocando que los electores se enfrentaran a cambios significativos a la hora de la elección sub nacional.

Ante esta situación, es necesario preguntarse sobre las posibles repercusiones de la introducción de este nuevo marco electoral en el aumento de voto nulo y blanco en las elecciones departamentales, sobre todo tomando en cuenta que el porcentaje de ciudadanos por departamento para elegir municipios tuvo mucha variación como se observa en el Cuadro 2.

\begin{tabular}{|c|c|c|}
\hline \multicolumn{3}{|c|}{$\begin{array}{l}\text { Cuadro 2. Relación entre aumento del voto inválido y porcentaje } \\
\text { de población habilitada para votar por municipio. }\end{array}$} \\
\hline DEPARTAMENTO & $\begin{array}{l}\text { Diferencia entre } \\
\text { promedio de } 2000 \text { y } \\
2005 \text { respecto a } 2010\end{array}$ & \begin{tabular}{lrr} 
Porcentaje & de & \multicolumn{2}{r}{ población } \\
habilitada para & votar por \\
municipio & \multicolumn{2}{c}{ según } \\
departamento &
\end{tabular} \\
\hline MONTEVIDEO & 282.07 & 79.1 \\
\hline CERRO LARGO & 225.28 & 18.0 \\
\hline CANELONES & 203.25 & 73.8 \\
\hline MALDONADO & 179.15 & 85.9 \\
\hline FLORES & 173.82 & 3.8 \\
\hline SORIANO & 137.55 & 25.3 \\
\hline ARTIGAS & 134.08 & 26.1 \\
\hline PAYSANDÚ & 126.21 & 10.9 \\
\hline SAN JOSÉ & 121.94 & 24.7 \\
\hline $\mathrm{ROCHA}$ & 121.75 & 37.4 \\
\hline COLONIA & 116.15 & 53.3 \\
\hline TACUAREMBÓ & 93.86 & 15.4 \\
\hline FLORIDA & 82.55 & 12.3 \\
\hline RIVERA & 72.77 & 11.0 \\
\hline RÍO NEGRO & 63.06 & 29.1 \\
\hline LAVALLEJA & 61.73 & 11.2 \\
\hline SALTO & 59.39 & 8.9 \\
\hline DURAZNO & 54.15 & 14.2 \\
\hline $\begin{array}{ll}\text { TREINTA } & Y \\
\text { TRES } & \end{array}$ & 30.83 & 10.8 \\
\hline
\end{tabular}

79 Para consultar sobre la puesta en marcha del proceso descentralizador ver Cardarello, Freigedo, Abraham y Vairo, 2010. 


\begin{tabular}{|l|l|l|}
\hline TOTAL & 79.06 & 29.01 \\
\hline
\end{tabular}

Fuente: Elaboración propia en base a datos de la Corte Electoral

De esta manera se puede establecer una relación del nivel de aumento de votos inválidos con el porcentaje de población habilitada por departamento para votar por municipio. En el Cuadro 2 se puede apreciar esta relación. Se destaca que en algunos casos, como el de Montevideo, Canelones y Maldonado el porcentaje de ciudadanos habilitados que votó por municipio fue muy alto (79.1, 73.8 y 85.9 respectivamente), como también fue significativo el aumento de votos inválidos a nivel departamental en estos lugares. Por su parte, se pueden apreciar casos en donde el porcentaje de población que votó por municipios fue bajo, siendo igualmente menor el aumento del voto blanco y nulo; ejemplo de esto es Salto y Treinta y Tres.

Sin embargo, también es posible observar casos en donde esta relación no se presenta de forma clara. Ejemplo de esto es Cerro Largo, en donde el porcentaje de aumento de los votos inválidos de las elecciones de 2010 fue de 225.28 por ciento, a pesar de que sólo un 18 por ciento del electorado estuvo habilitado para votar a nivel municipal. Lo contrario sucedió en Rio Negro, donde el voto inválido no aumentó tan significativamente (63.06 por ciento), pero fue uno de los departamentos en donde el porcentaje de habilitados para votar por municipios fue destacable si se compara con otros departamentos (29.1 por ciento).

Expuestos estos resultados y relaciones preliminares es pertinente evaluar sistemáticamente si el aumento del voto inválido fue consecuencia del cambio en el marco institucional, dada la incorporación del nuevo nivel de gobierno o, si responde a otras causas. El siguiente apartado está orientado a profundizar sobre la literatura que ha analizado este fenómeno $y$, específicamente se presentan las explicaciones que se han dado para el caso uruguayo en particular, con el objetivo de explorar posibles respuestas al fenómeno $y$, observar si dicha acumulación es útil para explicar lo acontecido en las elecciones sub nacionales de 2010 en Uruguay.

\section{ESTUDIOS SOBRE EL VOTO NULO Y BLANCO}

El estudio del voto nulo se ha realizado desde diferentes aproximaciones teóricas que acentúan distintos factores como determinantes de su aumento. Las perspectivas que se han utilizado para interpretar este fenómeno son tres: institucional, socioeconómica y política.

\section{a) El enfoque institucional}

Este enfoque enfatiza en el diseño institucional y considera como variables determinantes el voto obligatorio, la estructura de la boleta electoral, la complejidad 
del acto electoral, la existencia de lista abierta o cerrada para elegir representante, el equipo utilizado para que los ciudadanos voten y, la relación entre el voto de los electores y la proporción de asientos a ocupar en las cámaras legislativas.

La evidencia más destacada señala que los sistemas electorales con "voto obligatorio son efectivos para incrementar la participación, pero, cuando se presentan situaciones de desencanto o protesta, los votos válidos se traducen en un incremento de las boletas en blanco" (Hirczy, 1994: 74). Esto es debido a que en esos lugares, "(...) los votos blanco y nulos son equivalentes a la abstención en democracias con voto voluntario" (Lavareda, 1991: 40).

Por otra parte, también se ha encontrado que el voto inválido no sólo está asociado con el sufragio obligatorio, sino que combinado con otros elementos institucionales como las reglas electorales y el multipartidismo se genera su aumento (Power y Timmons, 1995: 819). Además, la estructura y la boleta electoral, así como la complejidad en el acto de sufragar también causan niveles altos de voto no válido (Power y Timmons, 1995: 819; Troumponis, 2010: 19; Power y Garand 2007).

\section{b) El enfoque socio-económico}

Este enfoque pone el punto de atención en el peso que tiene la estructura social y económica como determinante de este tipo de sufragio. Los factores que se consideran de relevancia son las características de las regiones o ciudades como la urbanización, la economía, el empleo, el nivel educativo, el analfabetismo, la migración y la desigualdad en el ingreso.

Se ha encontrado que las variables de mayor impacto en la invalidación del voto son el nivel de urbanización, la escolaridad, el analfabetismo y la migración. En la mayoría de los estudios, las primeras dos variables tienen una asociación negativa, mientras que el analfabetismo y la migración tienen una asociación positiva con el voto inválido, respectivamente. Sobre esto último, McAllister y Makkai (1993), al igual que Galatas (2008), encontraron que el sufragio inválido está determinado por el origen étnico.

Igualmente se ha demostrado que en las zonas donde existe una alta urbanización, mayores niveles de escolaridad e igualdad de ingresos, los porcentajes de voto inválido disminuyen en el electorado (Power y Garand, 2007). En relación específicamente con la urbanización, un estudio halló que en las zonas rurales hay más voto blanco que en las ciudades, lo cual se explica debido a que el control social y el conocimiento recíproco entre las personas de una comunidad pequeña, obligan a cumplir con el deber cívico, cuestión que no ocurre en las ciudades donde se encuentra un mayor porcentaje de abstencionistas (Zulfikarpasic, 2001). En ese sentido, en las zonas rurales el voto inválido puede ser un sinónimo de una abstención escondida, mientras que en las zonas urbanas es una expresión 
política.

Con respecto a la escolaridad, Power y Garand (2007) señalaron que altos niveles de educación están asociados con bajos niveles de voto nulo. Apoyando lo anterior, Galatas (2008) encontró que los votos anulados por error tienden a asociarse negativamente con bajos grados de instrucción. Ante esto, la relación esperada por este enfoque entre las variables voto nulo y escolaridad es negativa. Sin embargo, otros trabajos han demostrado que en situaciones de protesta, la escolaridad puede estar asociada positivamente con el porcentaje de votos anulados mostrando un claro signo de descontento político por parte de los electores más educados e interesados en la política (Cisneros 2013 y 2012b; Zulfikarpasic, 2001). Lo anterior da motivos para pensar que en determinadas circunstancias las personas que cuentan con una preparación académica por encima de la media se comportan de manera distinta a como usualmente ocurriría en situaciones normales.

c) El enfoque político

Finalmente, una tercera aproximación considera a las boletas blancas y anuladas como una forma de protesta política por parte de los ciudadanos. Desde esta perspectiva, el voto nulo es un hecho políticamente lógico, no es aislado, ni producto de la falta de habilidad para votar o de un sentimiento de apatía de parte del electorado.

Las variables determinantes que se consideran desde este enfoque son la competencia partidista, lo cerrado de las elecciones -poca distancia entre el primero y el segundo lugar-, la insatisfacción con las instituciones políticas, con la democracia o con la clase política, el desencanto después de transiciones democráticas, las movilizaciones de protesta que promuevan dicho tipo de sufragio $y$, el desalineamiento partidista.

Cuadro 3. Volatilidad partidaria en países europeos entre 1960 y 2000. 


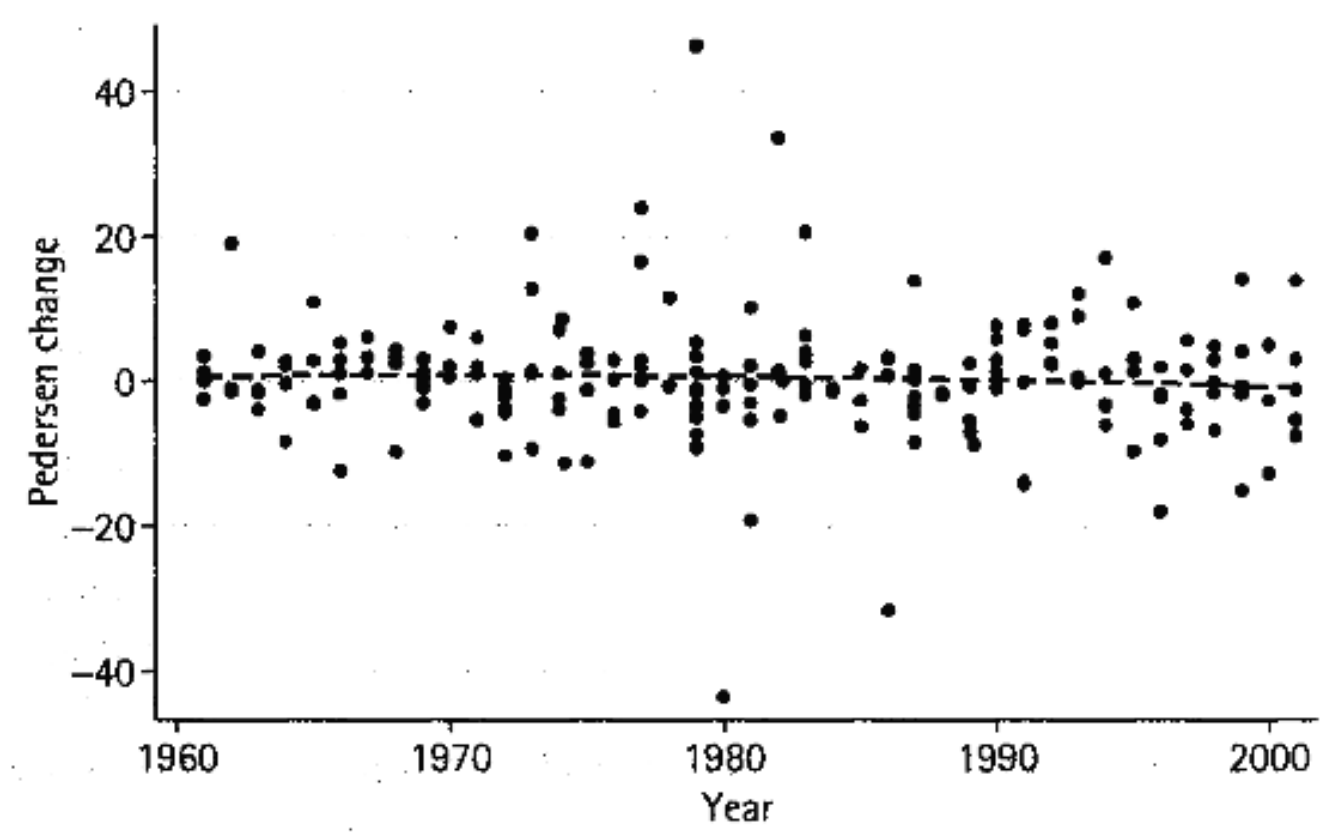

Fuente: Wren y Mcelwain (2007)

Sobre este último factor se destaca que en las democracias modernas los partidos políticos como instituciones de intermediación entre los ciudadanos y los representantes han ido perdiendo fuerza (Dalton, 1984 y 2000). Wren y Mcelwain (2007) señalan que se ha encontrado evidencia de un incremento en la inestabilidad de la relación entre votantes y partidos, acentuando lo que se conoce en la literatura como desalineamiento electoral. En ese sentido, Dalton (1984 y 2000) señala que la modernización en varios países ha traído consigo el surgimiento de un nuevo tipo de elector no identificado con ningún partido político, pero con alta escolaridad e interesado en los asuntos políticos, lo cual acarrea consecuencias importantes como el voto divido en apoyo a un partido para el congreso y a otro para la presidencia, la volatilidad (cambio de preferencia de partido) de elección a elección y, el incremento del voto inválido (Cisneros 2012 y 2012b) ${ }^{80}$. El siguiente cuadro muestra la volatilidad partidaria en países europeos entre 1960 y 2000, siendo evidente una alta inestabilidad del voto en relación a los partidos políticos medido a partir del Indice de Pedersen. Dalton (2000), por otro lado también muestra cómo en Alemania y Estados Unidos el porcentaje de votantes independientes ha crecido al interior del electorado generando cambios importantes en las decisiones de voto de los electores y en la estabilidad del voto hacia ciertos

80 Para el caso de México, Temkin et. al. (2008) encuentran que se ha asistido a un fenómeno de desalineamiento de los electores y aunque el sector de los apartidistas se ha incrementado (votantes escolarizados con alto interés en la política) y el grupo de votantes apolíticos es el que ha crecido mayoritariamente en las últimas décadas (electores con baja escolaridad y no interesados en la política). 
partidos.

De igual manera Wren y Mcelwain (2007), analizan cómo el voto total de los partidos ha declinado desde 1960, indicando en principio una fuerte presencia electoral de nuevos partidos, dado que las opciones existentes no son representativas de los electores. Asimismo, este cambio en la relación entre partidos y electores, ha derivado en la presencia de nuevos espacios de intermediación entre el ciudadano y el gobierno, muestra de esto es el surgimiento de movimientos sociales (Tarrow y Tilly, 2007) o de diferentes redes de advocacy (Keck y Sikking, 2007).

Pese a esta nueva configuración de los espacios de intermediación, los partidos siguen ocupando un lugar relevante en la política. Aunque sea posible observar de manera concreta como el desalineamiento electoral se ha ido incrementando en algunos países provocando en ocasiones el aumento de la protesta institucional y del voto nulo (Cisneros 2012b).

d) Los estudios del voto nulo y blanco en Uruguay

Antes de concluir, es necesario explorar las explicaciones que se han propuesto sobre el voto inválido en Uruguay para saber qué evidencia existe de la ocurrencia de este fenómeno. Como se mencionó, el alto porcentaje de voto nulo y blanco en Uruguay es una problemática reciente, por lo que la literatura al respecto es escasa. Una primera aproximación se puede encontrar en el estudio de Zuasnabar (2012) quien desde el enfoque político del voto nulo, analiza los cambios en la identificación partidaria (IP) en las elecciones departamentales de 2010. Sin embargo, como se observa en el Cuadro 4, para el caso de Uruguay es difícil hablar de un desalineamiento electoral fuerte ya que de los países de la región latinoamericana es el que muestra una de las menores tasas de electores no identificados con un partido político.

\section{Cuadro 4: Independientes 2006-2012 en América Latina y Uruguay}

\begin{tabular}{|l|l|l|l|l|l||}
\hline \hline & $\mathbf{2 0 0 6}$ & $\mathbf{2 0 0 8}$ & $\mathbf{2 0 1 0}$ & $\mathbf{2 0 1 2}$ & $\begin{array}{l}\text { Promedio } \\
\text { (2006- } \\
\text { 2012) }\end{array}$ \\
\hline Uruguay & 46,67 & 49,56 & 33,8 & 46,56 & 44,15 \\
\hline $\begin{array}{l}\text { Promedio } \\
\text { A.L. }\end{array}$ & 63,82 & 66,33 & 65,21 & 66,47 & 65,97 \\
\hline \hline
\end{tabular}

Sin embargo, una de las hipótesis que plantea Zuasnabar es que el alto grado de votos anulados y en blanco en Montevideo se debe a una caída de la IP frenteamplista. Pero, posteriormente esta hipótesis es rechazada por el mismo 
autor, ya que no encuentra variaciones importantes de la IP para este caso. Por tanto, es necesario encontrar otros factores que puedan explicar el fenómeno.

Este es el único estudio que además de plantear una posible hipótesis explicativa sobre las causas de los votos inválidos, trata de verificarla. Asimismo, hay otros trabajos que han planteado varias explicaciones al respecto pero ninguna ha sido puesta a prueba. Cardarello y Magri (2010) establecen como posible factor el desgaste de los ciudadanos ante el largo ciclo electoral que comenzó en octubre de 2009 con la elección nacional. Sin embargo, esta hipótesis no sería un factor explicativo sólido si se toma en cuenta que la elección anterior se presentaron los mismos factores ${ }^{81}$.

Otra de las causas que estos autores atribuyen al aumento del voto inválido se basa en la incorporación de las elecciones municipales. El hecho de votarse simultáneamente ambas elecciones pudo llevar a posibles efectos negativos y haber aumentado el número de votos anulados por el poco conocimiento ciudadano sobre este cambio en el marco institucional de las elecciones departamentales. Por último, al igual que Zuasnabar (2012) plantean la posibilidad de que exista un cambio en la identificación partidaria.

\section{MARCO TEÓRICO}

Para explicar qué factores están jugando en el aumento de los votos inválidos de las elecciones departamentales de 2010 se partirá de uno de las perspectivas de análisis de esta literatura: el enfoque institucional. Si bien en el estado del arte se señalan algunas variables institucionales como determinantes del aumento del sufragio nulo y blanco, éstas no son útiles para explicar el caso que aquí se pretende analizar. En primer lugar, no se han registrado cambios en cuanto a la obligatoriedad del voto, pues los ciudadanos están obligados a votar según el artículo 77 del Código civil, concibiendo al voto como un deber y no como un derecho desde hace más de una década. En segundo lugar, tampoco existieron cambios en la estructura de la boleta electoral ni en la existencia de listas abiertas o cerradas para elegir representantes 0 , en el equipo utilizado para que los ciudadanos voten.

De esta manera es que se retoma lo que otras investigaciones han advertido sobre el impacto que tiene el diseño institucional y las normas electorales en el comportamiento de los votantes. Y es que desde la primera mitad del siglo pasado, Duverger, tratando de explicar las determinantes del sistema de partidos, afirmó que en los sistemas electorales de mayoría de una sola vuelta se favorece un tipo

\footnotetext{
${ }^{81}$ La gran diferencia fue que en el 2005 las elecciones nacionales no tuvieron segunda vuelta (ballotage) debido a que el candidato del Frente Amplio obtuvo mayoría absoluta en la primera vuelta.
} 
de sistema bipartidista, mientras que en los sistemas de mayoría con segunda vuelta y en los sistemas de representación proporcional se favorece la formación de un multipartidismo (Duverger, citado en Cox, 2004, p. 32). La conclusión general de lo dicho por el autor francés es que el sistema electoral determina el sistema de partidos ${ }^{82}$.

En ese sentido, Cox también analiza el sistema electoral pero en cuatro vertientes distintas: las leyes y normas que regulan la forma en que los partidos hacen sus nominaciones; cómo votan los ciudadanos y realizan el escrutinio de los sufragios; la estructura de distrito y; cómo se transforman los votos escrutados en escaños. El autor afirma que el segundo, tercero y cuarto de esos aspectos están determinados por la ley electoral, mientras que el primero, por una combinación de la ley y del reglamento del partido (Cox, 2004, p. 58) ${ }^{83}$. Asimismo, señala que las elecciones involucran más que el acto de votar, dado que la votación está precedido por la competencia electoral, en la cual, las reglas definen la fórmula electoral, las magnitudes y límites distritales y, el tamaño de la asamblea, coordinando así la estrategia de elección de los votantes, partidos y candidatos sobre los votos y los escaños (Cox, 2004).

En relación con ello, Norris (2004) analiza específicamente los efectos del sistema electoral en la participación de los votantes. La autora retoma las dos perspectivas más importantes que estudian el tema: la elección racional y la perspectiva de la modernización cultural ${ }^{84}$. Sus resultados muestran que las reglas institucionales importan, pues la participación es maximizada en las elecciones con representación proporcional, con distritos pequeños, con competencia partidista y en elecciones

82 La Ley Duverger recibió bastantes críticas sobre todo de parte de la escuela del determinismo social. Actualmente, los que se oponen a esta última, señalan la importancia de la estructura social, pero consideran empero que ésta ejerce, sin excepción, una influencia importante e independiente (Cox, 2004, p. 34).

${ }^{83}$ Cox define al sistema electoral como "el conjunto de leyes y normas partidistas que rigen una competencia electoral entre los partidos y dentro de ellos" (2004, p. 58)

${ }^{84}$ La perspectiva de la elección racional sugiere que los incentivos de los ciudadanos a la participación en las elecciones puede ser entendido como un producto de: 1) los costos electorales de registrarse y votar, 2) las opciones partidistas disponibles para los electores, y 3 ) el grado en el que el voto determina la composición del parlamento y el gobierno. Downs (1973) afirma que la abstención es racional cuando los ciudadanos están convencidos de dos cosas: 1) de que los cambios de política que se producirán si resulta elegida la oposición no van a afectar a su renta de utilidad; 2) de que estos cambios pueden afectar a su renta de utilidad, pero que la probabilidad de que la eleven es exactamente igual a la de que se reduzca, es decir, que el cambio esperado es nulo. Por otro lado, la perspectiva de la modernización cultural señala que los patrones de participación cívica están asociados con los niveles de desarrollo humano, las características sociales como la educación, edad y clase y, actitudes como el sentido de eficacia política e interés. Desde esta perspectiva los hábitos de participación política responderán sólo ligeramente a los cambios en la ley electoral (Norris, 2004 , p. 257). 
presidenciales. Asimismo, argumenta que las leyes de voto obligatorio se encuentran asociadas con altos niveles de participación ${ }^{85}$.

Es claro entonces que el diseño institucional tiene un impacto en el comportamiento y en el resultado electoral. Ante ello, lo que se propone en este artículo es que el aumento del voto inválido estuvo asociado con la incorporación al juego electoral de un nuevo nivel de gobierno.

Y es que, como se mencionó, en las elecciones del 2010 se presentó un cambio significativo en el sistema electoral a nivel sub nacional con la inclusión de los municipios al sistema de gobierno, lo que determinó que ambas elecciones (departamental y municipal) se realizaran de manera conjunta. Sin embargo, en el país no todos los ciudadanos tuvieron la oportunidad de votar por autoridades municipales. La aprobación de la Ley de Descentralización y Participación Ciudadana estableció que solamente en aquellas localidades con más de 5000 habitantes se elegirían alcaldes. Por tanto, esto significó una variación muy importante en el porcentaje de ciudadanos que votaron por municipios, como ya se analizó en páginas anteriores.

De ahí que la primera hipótesis que guía este trabajo señale que el voto de protesta (asociado al nivel de escolaridad) ha incidido de manera importante en el voto inválido en las elecciones a nivel departamental del 2000 y 2005; sin embargo en las elecciones del 2010, si bien el sufragio de protesta siguió jugando un papel fundamental, el factor institucional también incidió de manera significativa para explicar en parte el aumento de este tipo de voto. En ese sentido, el aumento estará asociado principalmente a los cambios en el marco institucional y no a los factores de protesta.

Ahora bien, la hipótesis planteada responde a un análisis de votos inválidos en donde no se distingue entre votos blancos y nulos. Sin embargo, hay que tomar en cuenta que ambos tipos de sufragios no significan lo mismo y por tanto pueden responder a fenómenos diferentes. Para entender esto, es importante comentar brevemente el sistema de votación uruguayo.

Cuando el elector se encuentra en el cuarto de votación con un sobre en donde debe manifestar su preferencia electoral, las posibilidades de elección se reducen a cuatro: 1) poner la lista del partido de su preferencia y validar su voto; 2) dejar el sobre vacío y eso contabiliza como un voto en blanco; 3) cometer un error al ingresar la boleta al sobre y anular su voto; 4) introducir dentro del sobre, de manera voluntaria, un elemento anómalo que no sea una lista partidaria, lo cual implica también la anulación del voto.

${ }^{85}$ Como se señaló, se descarta que la legislación de voto obligatorio haya sido la causante del aumento de los votos nulos dado que este marco institucional se ha mantenido constante por lo menos en las últimas cuatro elecciones departamentales. 
Por lo tanto, el voto nulo implica que en el sobre de votación se incorporen anomalías sea por error o voluntarias, mientras que el voto en blanco significa que dentro del sobre de votación no se incorpora ninguna papeleta ni anomalía.

Así, mientras que el voto nulo puede responder tanto a errores en el cambio institucional como a un voto de protesta; el voto en blanco responde solamente al voto de protesta, ya que es difícil suponer que un elector cometa un error y deje el sobre de votación vacío sin tener la intención predeterminada de hacerlo, a menos que por desconocimiento de la norma lo haga.

En este sentido, la incorporación de un nuevo nivel de gobierno implica cambios en el diseño institucional electoral que puede arrojar como resultado que los electores se enfrenten ante nuevas opciones de votación que los lleve a cometer errores a la hora de emitir el voto, y así anaular el mismo. En el caso uruguayo el cambio está asociado a la creaciòn de los muncipios que conlleva que las elecciones a nivel subnacional (departamentales y municipales) se realicen de manera concurrente.

Como consecuencia de esto, la segunda hipótesis que se plantea en este artículo es que al separar el voto nulo del blanco, se apreciará que el voto nulo está significativamente relacionado con el cambio en el diseño institucional, mientras que el sufragio en blanco no necesariamente.

De esta manera, en lo que resta del artículo se intentarán comprobar las hipótesis planteadas, analizando la incidencia del cambio institucional y del voto de protesta en las elecciones de 2010 comparándolas con lo acontecido en los comicios de 2005.

\section{ANÁLISIS EMPÍRICO}

Para explicar el cambio en el voto inválido en Uruguay, es necesario construir una variable que permita medir la forma en que la inclusión del nuevo nivel de gobierno influyó en el aumento de este tipo de votos.

Así, se tomó en cuenta el porcentaje de personas que estaban habilitadas para votar en los nuevos municipios en cada departamento con el fin de comprobar si hubo una mayor proporción de votos inválidos en departamentos donde más porcentaje de la población votó por el tercer nivel de gobierno (Ver Cuadro 5) ${ }^{86}$.

Cuadro 5: Porcentaje habilitado para votar por municipios según Departamento

${ }^{86}$ Cabe destacar que por la naturaleza de nuestros datos lo que se propone realizar es un análisis agregado a nivel departamental y no un análisis de determinantes individuales, pues no se cuenta con la información que permita llevar a cabo esa tarea. 


\begin{tabular}{||l|l|l|l||}
\hline Maldonado & 85.9 & Tacuarembó & 15.4 \\
\hline Montevideo & 79.1 & Durazno & 14.2 \\
\hline Canelones & 73.8 & Florida & 12.3 \\
\hline Colonia & 53.3 & Lavalleja & 11.2 \\
\hline Rocha & 37.4 & Rivera & 11 \\
\hline Rio Negro & 29.1 & Paysandú & 10.9 \\
\hline Artigas & 26.1 & Treinta y Tres & 10.8 \\
\hline Soriano & 25.3 & Salto & 8.9 \\
\hline San José & 24.7 & Flores & 3.8 \\
\hline Cerro Largo & 18 & \multicolumn{2}{|l||}{} \\
\hline \hline
\end{tabular}

Para comprobar la fortaleza de esta variable se utilizarán distintas variables de control. Estas se seleccionaron tomando en cuenta los factores explicativos que la literatura retoma y la relación que éstas tienen con la variable dependiente y la independiente de interés. En relación con la variable de protesta se consideró el nivel de escolaridad por departamento, medido como el porcentaje de profesionales y técnicos universitarios por cada mil habitantes. El Cuadro 6 muestra dichos datos.

Por otro lado, también se seleccionaron dos variables de control en función de otro de los factores explicativos encontrados en la literatura: el nivel socioeconómico. Para este cometido se controló por la variable de zonas urbanas y producto interno bruto (PIB) per cápita, la tasa de desempleo y el índice de gini por cada uno de los departamentos. Asimismo, se utilizó un control sociodemográfico, al incluirse una variable de porcentaje de población femenina.

\begin{tabular}{|c|c|c|c|}
\hline \multicolumn{4}{|c|}{$\begin{array}{l}\text { Cuadro 6: Profesionales y técnicos universitarios cada } 1000 \\
\text { habitantes por Departamento }\end{array}$} \\
\hline Montevideo & 48.1 & Florida & 10.1 \\
\hline Salto & 15.2 & Lavalleja & 10 \\
\hline Maldonado & 14.9 & Rio Negro & 9.8 \\
\hline Paysandú & 13.7 & Rocha & 9.8 \\
\hline Canelones & 12.9 & Durazno & 8.9 \\
\hline Colonia & 12.8 & Artigas & 8.1 \\
\hline Flores & 12.6 & San José & 8.1 \\
\hline
\end{tabular}




\begin{tabular}{|l|l|l|l||}
\hline Soriano & 11.6 & Cerro Largo & 7.9 \\
\hline Treinta y Tres & 11.5 & \multirow{2}{*}{ Rivera } & 7.8 \\
\cline { 1 - 2 } Tacuarembó & 11.0 & & \\
\hline \multicolumn{2}{|l||}{ Fuente: Diagnóstico de cohesión territorial para Uruguay, 2011 } \\
\hline
\end{tabular}

Con la información así reunida se construyeron cuatro modelos de regresión, dos para comparar los resultados de los votos inválidos de 2005 con los de 2010, y dos más en los que se desagregó la variable dependiente de 2010 en sufragios en blanco y anulados para averiguar si las variables incluidas en el modelo explican de diferente manera los diferentes tipos de voto.

Asimismo, se recurrió al método de regresión robusta debido a que ayuda a "proporcionar estimadores sensibles dada la presencia de datos anómalos y a la no normalidad y homocedasticidad del error aleatorio" (Escobar, et. al., 2009) pues el número de casos que se están considerando en este trabajo son sólo 19 -número de departamentos que existen en Uruguay-, lo cual no permite un comportamiento normal de los datos analizados, por lo que se recurre al método señalado.

Como se observa en el Cuadro 7, los resultados muestran que en 2005 el voto inválido departamental estuvo asociado significativamente con la escolaridad y con las zonas urbanas. Con respecto a esta última, la asociación fue negativa, mientras que la relación con la escolaridad fue positiva, lo cual significa que a nivel departamental los sectores con mayores niveles de escolaridad anularon su sufragio mayoritariamente. Dicha relación puede interpretarse como una señal de protesta o de inconformidad con la política en ese nivel de gobierno, pues, siguiendo la literatura sobre el tema se señala que en lo general la asociación entre escolaridad y voto nulo es negativa, siendo positiva sólo en situaciones de protesta o desencanto con las instituciones políticas (Power y Garand, 2007). Lo anterior contradice los hallazgos señalados por parte de la literatura previa del enfoque socio-económico que enfatizaba una asociación negativa entre ambas variables.

\begin{tabular}{|l|l|l|}
\hline $\begin{array}{l}\text { Cuadro 7: Regresión líneal múltiple } \\
\text { Comparación de determinantes del voto inválido } \\
\text { departamental } 2005 \text { y } 2010\end{array}$ \\
\hline $\begin{array}{l}\text { Variable dependiente } \\
\text { voto inválido } \\
\text { departamental }\end{array}$ & 2005 & 2010 \\
\hline \hline Escolaridad & $\begin{array}{l}0.014 * * \\
{[0.005]}\end{array}$ & $\begin{array}{l}-0.012 \\
{[0.019]}\end{array}$ \\
\hline Diseño institucional & -- & $0.045^{*}$ \\
\hline
\end{tabular}




\begin{tabular}{|c|c|c|}
\hline & & {$[0.021]$} \\
\hline Mujeres & $\begin{array}{l}0.141 \\
{[0.195]}\end{array}$ & $\begin{array}{l}1.055^{*} \\
{[0.563]}\end{array}$ \\
\hline Urbano & $\begin{array}{l}-0.081^{*} \\
{[0.038]}\end{array}$ & $\begin{array}{l}-0.031 \\
{[0.140]}\end{array}$ \\
\hline Índice de gini & $\begin{array}{l}0.075 \\
{[0.050]} \\
\end{array}$ & \begin{tabular}{|l|}
0.237 \\
{$[0.143]$} \\
\end{tabular} \\
\hline PIB pc & $\begin{array}{l}0.006 \\
{[0.008]} \\
\end{array}$ & $\begin{array}{l}0.006 \\
{[.028]} \\
\end{array}$ \\
\hline Voto inválido 2000 & $\begin{array}{l}0.757^{* *} \\
{[0.208]} \\
\end{array}$ & -- \\
\hline Voto inválido 2005 & -- & $\begin{array}{l}1.380 * \\
{[0.674]} \\
\end{array}$ \\
\hline Constante & $\begin{array}{l}-0.072 \\
{[0.086]}\end{array}$ & $\begin{array}{l}-0.647 * * \\
{[0.286]} \\
\end{array}$ \\
\hline Observaciones & 19 & 19 \\
\hline R cuadrada ajustada & 0.663 & 0.799 \\
\hline Prob $>\mathrm{F}$ & $F(6,12)=6.91 * * *$ & $\begin{array}{l}\mathrm{F} \quad(7,11)= \\
11.22 * * *\end{array}$ \\
\hline \multicolumn{3}{|c|}{$\begin{array}{l}\text { Error estándar entre corchetes } \\
* * * p<0.01, * * p<0.05, * p<0.1 \\
\text { Fuente: Elaboración propia con datos de la Corte Electoral y del } \\
\text { documento Diagnóstico de cohesión territorial para Uruguay, } 2011 .\end{array}$} \\
\hline
\end{tabular}

Cabe destacar que de lo anterior no pueden inferirse comportamientos individuales, como por ejemplo, señalar que las personas con mayores estudios anularon su voto con mayor probabilidad pues se caería en la falacia ecológica que consiste en inferir de datos agregados comportamientos individuales (Welzel e Inglehart, 2007). Lo que se puede afirmar con certeza es que en los departamentos con mayor escolaridad promedio se tendió a invalidar el sufragio en mayor medida.

Ahora bien, con respecto a la elección de 2010, los indicadores económicos no mostraron una asociación significativa con el sufragio inválido. Se destaca que el sector femenino estuvo relacionado positivamente con el voto nulo, mostrando además un coeficiente de asociación fuerte en comparación con las demás variables. Lo anterior significa que a nivel departamental las mujeres tuvieron una tendencia mayoritaria a anular el voto en estos comicios.

En cuanto a la escolaridad, en 2010 la relación establecida con la variable dependiente no fue significativa -a diferencia de 2005 donde esta variable sí fue 
significativa y forjó una relación positiva. Finalmente, en relación con la variable de interés (diseño institucional), ésta resultó significativa y forjó una relación positiva con los votos inválidos, por lo que se puede afirmar que el aumento de este tipo de votos en estas elecciones tuvo como causa fundamental la incorporación de un nuevo nivel de gobierno al juego electoral.

Por lo anterior es que a diferencia de 2005 donde se encontró la existencia de un voto de protesta debido a la asociación positiva entre la anulación del sufragio y la escolaridad, en 2010 el aumento del voto inválido no se debió a un voto de protesta sino a la incorporación de factores institucionales como se había planteado en un principio.

Ahora bien, a pesar de lo señalado, cuando se desagrega el voto inválido en nulo y blanco como se aprecia en el modelo de regresión del Cuadro $8^{87}$, la variable de interés muestra de nuevo su importancia afectando tanto al sufragio en blanco como al anulado. En ambos casos la relación resultó positiva, repercutiendo de manera más importante en el voto blanco. Esta situación, si bien puede parece extraña debe de ponerse en relación con los demás resultados.

Respecto al voto blanco, no sólo la incorporación de un nuevo nivel de gobierno hizo que los ciudadanos acudieran a las urnas y no votaran, sino que además, este comportamiento agregado ocurrió en combinación con la alta escolaridad y con los sectores de mayores recursos económicos a nivel departamental, pues tanto el ingreso promedio, la desigualdad y la tasa de desempleo tuvieron una asociación significativa con las boletas blancas. Las primeras dos variables en sentido positivo y la tercera en dirección opuesta.

Estas asociaciones muestran que si bien el ciudadano tuvo la posibilidad de votar por autoridades municipales, prefirió dejar la boleta en blanco dejando entrever al menos dos posibilidades: 1) que no se quisiera votar dado el desconocimiento de los candidatos y la nueva regulación o; 2) que decidiera no sufragar en señal de protesta por la falta de opciones políticas.

Si bien este resultado abre más preguntas en vez de brindar una respuesta certera, deja ver que el estudio de este tipo de voto suele ser complejo, sobre todo cuando existen legislaciones como el voto obligatorio, la cual, en ocasiones disfraza la abstención de votos inválidos. A pesar de ello, se puede afirmar con certeza que el nuevo marco institucional repercutió en el comportamiento de los votantes a nivel departamental llevándolos a sufragar en blanco en lugar de votar por un partido político.

${ }^{87}$ En los resultados del modelo de regresión robusta el análisis excluyó al departamento de Rocha de la explicación del voto nulo, mientras que en el modelo que explica al voto blanco excluyó el departamento de Canelones, logrando con esto un mejor ajuste en los resultados. 


\begin{tabular}{|c|c|c|}
\hline \multicolumn{3}{|c|}{$\begin{array}{l}\text { Cuadro 8: Regresión robusta } \\
\text { Comparación de determinantes del voto blanco y nulo } \\
\text { departamental } 2010\end{array}$} \\
\hline $\begin{array}{l}\text { Variable dependiente } \\
\text { voto blanco y nulo } \\
\text { departamental }\end{array}$ & Voto blanco & Voto nulo \\
\hline Escolaridad & $\begin{array}{l}0.011^{* * *} \\
{[0.002]}\end{array}$ & $\begin{array}{l}0.085^{* * *} \\
{[0.016]}\end{array}$ \\
\hline Diseño institucional & $\begin{array}{l}0.008^{* *} \\
{[0.003]}\end{array}$ & \begin{tabular}{|l|}
$0.005^{*}$ \\
{$[0.002]$} \\
\end{tabular} \\
\hline Mujeres & $\begin{array}{l}0.272^{* *} \\
{[0.085]}\end{array}$ & $\begin{array}{l}-0.102 \\
{[0.105]}\end{array}$ \\
\hline Urbano & $\begin{array}{l}0.004 \\
{[0.019]}\end{array}$ & $\begin{array}{l}-0.039 * * \\
{[0.017]}\end{array}$ \\
\hline Índice de gini & $\begin{array}{l}0.372 * * * \\
{[0.021]}\end{array}$ & $\begin{array}{l}-0.008 \\
{[0.019]}\end{array}$ \\
\hline Tasa de desempleo & $\begin{array}{l}-0.799 * * * \\
{[0.042]}\end{array}$ & $\begin{array}{l}0.027 \\
{[0.037]}\end{array}$ \\
\hline PIB pc & $\begin{array}{l}0.038 * * * \\
{[0.004]}\end{array}$ & $\begin{array}{l}-0.002 \\
{[0.007]}\end{array}$ \\
\hline Constante & $\begin{array}{l}-0.554 * * * \\
{[0.043]} \\
\end{array}$ & $\begin{array}{l}0.112 \\
{[0.108]} \\
\end{array}$ \\
\hline Observaciones & 18 & 18 \\
\hline Prob $>\mathrm{F}$ & $\begin{array}{l}\mathrm{F} \quad(7,10)= \\
272.95^{* * *}\end{array}$ & \begin{tabular}{|l}
$\mathrm{F} \quad(7,10)$ \\
$43.20 * * *$
\end{tabular} \\
\hline \multicolumn{3}{|c|}{$\begin{array}{l}\text { Error estándar entre corchetes } \\
* * * p<0.01, * * \mathrm{p}<0.05, * \mathrm{p}<0.1 \\
\text { Fuente: Elaboración propia con datos de la Corte Electoral y del } \\
\text { documento Diagnóstico de cohesión territorial para Uruguay, } 2011\end{array}$} \\
\hline
\end{tabular}

Con respecto al voto nulo, los hallazgos muestran, como se mencionó, que la incorporación de un nuevo nivel de gobierno tuvo un impacto significativo y positivo en el porcentaje de votos nulos. Asimismo, la escolaridad también forjó una asociación positiva y significativa, dejando entrever nuevamente la existencia de un sufragio de protesta, es decir, de un voto nulificado voluntariamente y no por error.

Por último, las viviendas urbanas estuvieron asociadas negativamente con este tipo de voto, lo cual es un hallazgo esperado de la literatura debido a que en los sitios con esa característica suele haber menos voto nulo. Así también vale la pena destacar que la anulación del sufragio a diferencia del voto blanco, no mostró 
asociación con ninguna de las variables económicas, siendo importante resaltar que a pesar de que el sector escolarizado anuló el sufragio, no ocurrió de la misma manera que con el voto blanco, donde los departamentos con mayores recursos económicos también dejaron la boleta vacía.

\section{CONCLUSIONES}

El aumento de los votos anulados y blancos en las elecciones departamentales del 2010 en Uruguay fue una hecho inédito en un país caracterizado por los altos niveles de cultura cívica y el respaldo ciudadano a la democracia. Este trabajo intentó ser un primer acercamiento a la explicación de dicho fenómeno que ha sido poco explorado por la literatura existente.

Para este cometido, primero se plantearon las respuestas que desde la literatura se dan al problema, acentuando los factores institucionales, económicos y políticos. Sin embargo, tomando en cuenta la realidad del sistema electoral uruguayo a nivel sub nacional, y especialmente a partir de la incorporación de un nuevo nivel de gobierno en las elecciones; la literatura no respondía consistentemente a este nuevo fenómeno, pues, no existía una referencia teórica ni empírica que pudiera explicar la consecuencia de una modificación en el diseño instititucional. Ante ello, se recurrió a bibliografía de la ciencia política contemporánea que explica la incidencia de los cambios institucionales en el comportamiento de los votantes. Así, se construyeron una serie de hipótesis que vincularon los factores institucionales con el fenómeno de los votos inválidos, intentando realizar un aporte para la discusión de estos casos.

Los hallazgos más relevantes del análisis demuestran que a nivel departamental el aumento del voto inválido agregado (es decir blanco y nulo en conjunto) para las elecciones de 2010 estuvo asociado a los cambios institucionales, es decir, a la incorporación de un nuevo nivel de gobierno, mientras que en 2005 los niveles de voto nulo se asociaron con la alta escolaridad departamental, lo cual es indicativo de un voto de protesta política.

Teniendo en cuenta los resultados del análisis en 2010, no se puede entender el aumento del voto nulo como consecuencia de un desalineamiento electoral (Zuasnabar, 2011) o como una disminución en la confianza institucional (Temkin y Salazar 2007). Asimismo, no se puede concluir que el voto inválido se relacione únicamente con un voto de protesta o inconformidad con la gestión de los gobiernos departamentales a nivel agregado. Aunque, para una confirmación consistente de estos planteamientos es necesario contrarrestar este análisis con uno a nivel individual para saber si dichas afirmaciones tiene sustento, aunque a nivel agregado estas hayan quedado descartadas. 
Por otro lado, la segunda de las hipótesis puestas a prueba en este artículo también se confirmó, pues al estudiar los votos inválidos agregados, el cambio en el diseño institucional tuvo impacto significativo; cuando se analizaron ambos tipos de votos por separado se comprobó igualmente que la incorporación de un nuevo nivel de gobierno tuvo como consecuencia el aumento del voto blanco y nulo, respectivamente. Entre las posibles causas que explican esta relación, se puede suponer que la falta de información por parte del electorado ante los cambios en el diseño institucional electoral pudo llevar a confusiones y a errores a la hora de emitir el voto. De todas formas, también existe una relación significativa con el nivel de escolaridad, por lo que a su vez se puede entender el aumento del voto nulo y blanco como consecuencia de un signo de protesta aunque en el agregado esto no se haya comprobado.

En suma, lo que se demostró aquí fue que el aumento del voto inválido en las elecciones del 2010 no estuvo asociado a un voto de protesta por parte de los ciudadanos, siendo significativa la variable de inclusión de un nuevo nivel de gobierno tanto en el agregado de votos inválidos como al momento de separarlos en sufragios nulos y blancos.

$Y$ es que al tratarse de un fenómeno reciente, que no ha sido estudiado en profundidad, los hallazgos aquí presentados son un primer aporte para entender un problema poco recurrente. De cualquier manera, quedan abiertas preguntas que deben ser profundizadas en posteriores estudios. En especial, se debe comprobar que el voto de protesta esté asociado efectivamente a la inconformidad con la gestión de las Intendencias Departamentales y; que el aumento del voto nulo se debe a la falta de información sobre los cambios en el diseño institucional electoral ${ }^{88}$.

Por último, se debe resaltar que este estudio puede ser un aporte para entender el comportamiento electoral sub nacional en dos sentidos. En primer lugar, si bien en el caso uruguayo se analizó la incorporación de un nuevo nivel de gobierno al sistema electoral sub nacional; se debe tomar en consideración para otros casos, cómo los cambios en el diseño institucional en un nivel de gobierno pueden afectar el comportamiento del electorado sobre otros niveles de gobierno.

En segundo lugar, este artículo también es un aporte para entender uno de los asuntos más complejos a la hora de analizar los gobiernos sub nacionales: que los individuos logren distinguir las competencias de cada nivel (y rama de gobierno) y a través de su voto, castigar -despedir- a los malos gobernantes. Analizar las diferencias en el comportamiento del porcentaje de votos inválidos en niveles de gobierno disímiles contribuye a la discusión sobre el tema.

${ }^{88}$ Cabe señalar que este artículo solamente se centró en el análisis de los resultados a nivel departamental y no en las causas que explican el alto grado de abstencionismo en las elecciones municipales. 


\section{Bibliografía}

- Cardarello, Antonio y Magri, Altair (2010) "Elecciones Departamentales 2010: consecuencias imprevistas, resultados esperados" en Daniel Buquet y Niki Johnson (coord.): Del cambio a la continuidad. Ciclo electoral 2009-10. Págs. 295-324. Ediciones de la Banda Oriental - Instituto de Ciencia Política de la Facultad de Ciencias Sociales. Universidad de la República, Montevideo. ISBN 9789974495043

- Cardarello, Antonio; Freigedo, Martín; Abraham, Manuela; Vairo, Daniela (2010) "Mucho ruido... y algunas nueces. Los vaivenes de la descentralización en la administración Vázquez (2005-2010)". En Pedro Narbondo y María Ester Mancebo (coord.) Reforma del Estado y políticas públicas de la Administración Vázquez: acumulaciones, conflictos y desafíos. Ed: Fin de Siglo. Montevideo.

- Cisneros, Isaac (2013), "Movilización, escolaridad y voto nulo. La elección federal de 2009 en México", Política y gobierno, vol. XX, núm. 1, pp. 39-78, ISSN-1665-2037

(2012), "La movilización por la anulación del voto en 2009: una nueva forma de protesta política", Revista Mexicana de Ciencias Políticas y Sociales, número 215 , mayo-agosto.

- _ (2012b), "El efecto de la movilización anulista en el voto nulo de la elección para Diputados Federales de 2009 en México", Tesis de maestría, Facultad Latinoamericana de Ciencias Sociales, pp. 65-86.

- Cox, Gary (2004), "La coordinación estratégica de los sistemas electorales del mundo: hacer que los votos cuenten", Gedisa, Barcelona.

- Dalton, Russell (1984), "Cognitive mobilization and partisan dealignment in advanced industrial democracies", The Journal of Politics, vol. 1, núm. 1, pp. 264-284.

- Downs, Anthony (1973). "Teoría Económica de la Democracia". Madrid: Aguilar.

- _ (2000), "The decline of party identifications" en Russell Dalton y Martin Wattenberg (eds.), Parties without partisans. Political change in advanced industrial democracies, New York, Oxford University Press, pp. 19-34.

- Escobar, Modesto, et al. (2009), "Análisis de datos con STATA", Cuadernos metodológicos, España.

- Freigedo, Martín y Lorenzoni, Miguel (2011). "La capital se vistió de blanco. Elecciones departamentales y municipales en el Departamento de Montevideo". En Antonio Cardarello y Altair Magri (coord.) Cambios Certezas e Incertidumbres. Elecciones departamentales y municipales 2010. Departamento de Ciencia Política, Facultad de Ciencias Sociales, Universidad de la República, 
Congreso Nacional de Intendentes. AEXCID. Montevideo.

- Galatas, Steven (2008), "None of the above? casting blank ballots in Ontario provincial elections", Politics and policy, vol. 36, núm. 3, pp. 448-473.

- Hirczy, Wolfgang (1994), "The impact of mandatory voting laws on turnout: a quasi-experimental approach", Electoral Studies, vol. 13, núm. 1, pp. 64-76.

- Keck, Margaret E. y Sikkink, Kathrin (2007) "Transnational Contentious Politics" en Kesselman, Mark (coord). The politics of globalization. Boston, Mass.: Houghton Mifflin

- Lavareda, José Antônio (1991), "A democracia nas urnas: o processo partidário electoral brasileiro". Brasil, Río Fundo Editora.

- López, Santiago (2007), "¿Cuán locales son las elecciones municipales en Uruguay? Estimando independencia de resultados y comportamientos electorales" en Revista Uruguaya de Ciencia Política, número 16. Instituto de Ciencia Política, FCS. Montevideo.

- Magri, Altair y Freigedo, Martín (2010) "Municipales 2010: ¿elecciones de segundo orden?" en Daniel Buquet y Niki Johnson (coord.): Del cambio a la continuidad. Ciclo electoral 2009-10. Págs. 325-369. Ediciones de la Banda Oriental - Instituto de Ciencia Política de la Facultad de Ciencias Sociales. Universidad de la República, Montevideo. ISBN 9789974495043.

- McAllister, Ian y Toni Makkai (1993), "Institutions, society or protest? Explaining invalid votes in Australian elections", Electoral Studies, vol. 12, núm. 1, marzo, pp. 23-40.

- Norris, Pippa (2004), "Electoral engineering. Voting rules and political behavior", New York, Cambridge Univesity Press.

- Power, Timothy y Roberts Timmons (1995), "Compulsory Voting, Invalid Ballots, and Abstention in Brazil", Political Research Quarterly, vol. 48, núm. 4, pp. 795826.

- Power, Timothy y James Garand (2007), "Determinants of invalid voting in Latin America", Electoral Studies, vol. 26, pp. 432-444.

- Rodriguez, Adrián (2011) "Diagnóstico de cohesión territorial para Uruguay", Informe del Programa Uruguay Integra, Oficina de Planeamiento y Presupuesto. Montevideo.

- Salazar, Rodrigo y Benjamín Temkin (2007), "Abstencionismo, escolaridad y confianza en las instituciones. Las elecciones federales de 2003 en México", Política y gobierno, vol. XIV, núm. 1, primer semestre, pp. 5-42.

- Tarrow, Sidney y Tilly, Charles (2007) "Contentious politics". Series en (Political Science / Sociology). Boulder, Colo.: Paradigm Publishers. 
- Temkin, Benjamín, et al. (2008), "Explorando el <<apartidismo>> en México: ¿Apartidistas o apolíticos?", América Latina hoy, núm. 50, pp. 119-145.

- The Americas Barometer by the Latin American Public Opinion Project (LAPOP), www.LapopSurveys.org.

- Verba, Sydney y Norman H. Nie (1972), "Participation in America. Poitical democracy and social inequality", Nueva York, Harper \& Row

- Welzel, Christian; Inglehart, Ronald (2007), "Mass beliefs and democratic institutions" en Oxford Handbook of Comparative Politics, London.

- Wren, K., Keneth Mcelwain (2007), "Voters and parties: realignment or dealignment in the party-voter nexus, en Oxford Handbook of Comparative Politics, London, pp. 555-581.

- Zuasnabar, Ignacio (2012) "Las elecciones departamentales 2010 bajo la lupa de la identificación partidaria" en Luis Eduardo González, Federico Irazábal, Pablo Mieres e Ignacio Zuasnabar: El voto en Uruguay 2009-2010. Konrad Adenower y Universidad Católica del Uruguay. Montevideo.

- Zulfikarpasic, Adélaïde (2001), "Le vote blanc: abstention civique ou expresión politique?", Revue francaise de science politique, núm. 1-2, pp. 247-268.

\section{Fuentes de información}

www. corteelectoral.gub.uy 